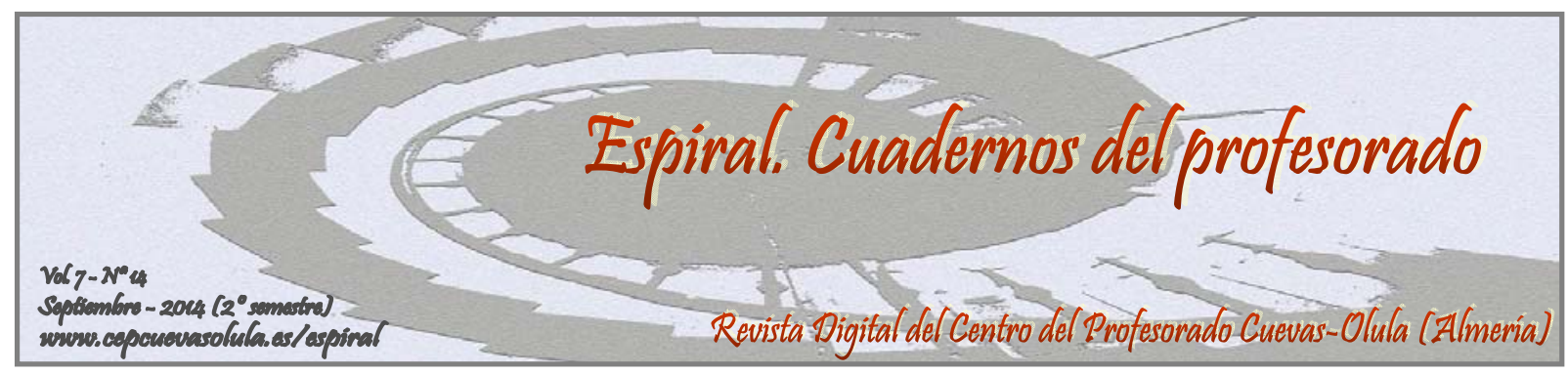

\title{
ESTADO DE ÁNIMO, AUTOCONFIANZA Y ANSIEDAD PRECOMPETITIVA EN TIRO DEPORTIVO
}

\section{MOOD, SELF-CONFIDENCE AND PRE-COMPETITIVE ANXIETY IN SPORT SHOOTING}

\section{José Manuel Rodríguez-Gómez ${ }^{(1)}$ y Antonio Granero-Gallegos ${ }^{(2)}$}

(1) Universidad Autónoma de Madrid, España

(2) Consejería de Educación, Cultura y Deporte de la Junta de Andalucía, España

RESUMEN: El objetivo de este estudio fue analizar los niveles de estado de ánimo y ansiedad precompetitiva, según el nivel de los deportistas. Han participado un total de 183 deportistas con edades entre 26 y 59 años $(M=41.14 ; D T=6.43) ; 179$ eran hombres y solamente cuatro mujeres. Se utilizaron los siguientes instrumentos: Competitive State Anxiety Inventory-2 y Profile of Mood States. Se realizó un análisis multivariante de la varianza y se realizó el cálculo de la dispersión de los impactos a través de un programa informático diseñado para tal efecto. Se halló una relación directamente lineal entre el rendimiento, el vigor y la autoconfianza; y una relación inversamente lineal entre éste y la ansiedad somática. En las variables del estado de ánimo se encontró el denominado "perfil iceberg". Respecto a la dispersión de los impactos se observó una descentralización con tendencia hacia la izquierda y hacia abajo, siendo mayor la dispersión a medida que desciende la categoría. Por otra parte los tiradores diestros agrupan a la izquierda y los zurdos a la derecha.

Palabras clave: Competición, tirador, vigor, ansiedad somática, ansiedad cognitive

ABSTRACT: The aim of this study was to analyze the levels of pre-competitive mood and anxiety, according to the level of athletes. They involved a total of 183 athletes aged between 26 and 59 years old $(M=41.14, S D=6.43) ; 179$ were men and only four were women. The following instruments were used: Competitive State Anxiety Inventory-2 and Profile of Mood States. A multivariate analysis of variance was performed and the calculation of the dispersion of the impact is performed using a computer program designed for this purpose. One directly linear relationship between yield, vigour and self-confidence was found; and an inverse linear relationship between it and somatic anxiety. In the mood variables called "iceberg profile" was not found. Regarding the dispersion of the impact prone decentralization and leftward down was observed, the dispersion being higher as the class descends. Moreover the right-handed shooters grouped on the left and right handed.

Key words: Competition, shooter, vigour, somatic anxiety, cognitive anxiety

Rodríguez-Gómez, J. M. y Granero-Gallegos, A. (2014). Estado de ánimo, autoconfianza y ansiedad precompetitiva en tiro deportivo. Espiral. Cuadernos del Profesorado, 7(14), 13-23. Disponible en: http://www.cepcuevasolula.es/espiral.

Fecha de recepción: 12/02/2014

Fecha de aceptación: 20/06/2014
Enviar correspondencia a: pinodeloro@hotmail.com 


\section{1.- INTRODUCCIÓN}

La importancia de las relaciones entre el rendimiento deportivo y el estado de ánimo ha sido objeto de estudio en numerosas de investigaciones, tanto a nivel nacional como internacional, sobre todo con estudiantes universitarios (Balaguer, Fuentes, Melia, García-Merita \& Pérez, 1994; Rey, Blasco \& Borrás, 2000). Asimismo, se han realizado trabajos en el campo de la recuperación de lesiones deportivas (Abenza, Olmedilla, Ortega, Ato, \& García-Mas, 2010). En el ámbito del rendimiento deportivo también se ha investigado en jóvenes futbolistas federados (Andrade, Arce, Armental, Rodríguez, \& De Francisco, 2008; Arruza, Arribas, Otaegi, Gonzales, Irazusta, \& Ruiz, 2011; Arruza, Balagué \& Arrieta, 1998), judocas de élite (Arruza, 1995; Torres-Luque, HernándezGarcía, Olmedilla, Ortega, \& Garatachea, 2013), fisicoculturistas (Arbinaga, 2013), en comparativa entre deportes (Moreno, Parrado, \& Capdevila, 2013), así como en jugadores de futbol pertenecientes a la segunda división española (De la Vega, Ruiz Barquín, García Adrianzén, \& Del Valle, 2011; De la Vega, Ruiz Barquín, García-Más, Balagué, Olmedilla, \& Del Valle 2008).

Uno de los instrumentos más utilizados en investigación y práctico en la determinación y estudio de los estados de ánimo óptimos o facilitadores de rendimiento en deportistas es el denominado Profile of Mood States (POMS) (Cox, 2008). Este instrumento plantea la existencia de dos grandes factores para interpretar las principales dimensiones de la estructura afectiva (Watson \& Clark, 1994, 1997); por un lado, el afecto o estado de ánimo positivo y, por otro, el afecto negativo. Son estas dos grandes dimensiones del estado de ánimo las que han sido desarrolladas con este instrumento (McNair, Lorr \& Dropleman, 1971) que ha llegado al deporte de la mano de Morgan (1980a), a partir de su Modelo de Salud Mental (Mental Health Model). Según este último autor, el estado de ánimo positivo y el éxito deportivo correlacionan de forma significativa. Las puntuaciones en los distintos factores del POMS han permitido a este autor identificar, incluso, un patrón de estado de ánimo característico de los deportistas con más éxito, al que denominó perfil iceberg. Esta denominación se debe al hecho que sus valores en ansiedad, depresión, ira y fatiga suelen situarse por debajo de la media de la poblacional; mientras que sus puntuaciones en vigor suelen ser superiores a dicha norma (Morgan, 1980a, 1980b; O'Connor, 2006). En este sentido, el perfil de estado de ánimo predispondría al jugador a obtener un elevado rendimiento competitivo que se correspondería con un estado de ánimo óptimo (De la Vega et al., 2008). Por otra parte, Cox (2008) observó como los deportistas de mayor éxito obtenían un perfil de estados de ánimo más saludable, frente a aquellos menos exitosos o con una población control.

En cuanto a la ansiedad, Jaenes (2000), en función de la estabilidad de la ansiedad, distinguía entre ansiedad rasgo (ser), como la tendencia personal a tener ansiedad en la mayoría de situaciones de la vida cotidiana, y ansiedad estado (estar), como la manifestada por una persona en una situación concreta. Desde esta perspectiva Ruiz-Barquín y Rodríguez (2013), en un estudio con tiradores deportivo, modalidad de Tiro Práctico, encuentran correlaciones inversas y negativas entre la ansiedad estado y el rendimiento.

Desde una aproximación multidimensional, Martens, Burton, Vealey, Bump, y Smith (1990) diferenciaron tres sistemas de respuesta o dimensiones en la ansiedad precompetitiva. Las preocupaciones respecto a la ejecución y falta de habilidad para concentrarse son conocidas como ansiedad cognitiva (Eysenck \& Calvo, 1992; Liebert \& Morris, 1967). A esta dimensión pertenecen las experiencias de miedo, pánico, alarma, inquietud, aprensión, obsesiones y pensamientos intrusivos de tipo catastrófico. La ansiedad somática es la percepción de los síntomas corporales causados por la activación del sistema nervioso autónomo, tales como aceleración del ritmo cardíaco, sudoración, etc., en situaciones de ansiedad cognitiva (Cheng, Hardy, \& Markland, 2009; Hardy, Jones, \& Gould, 1996; Martens et al., 1990). Es la dimensión de ansiedad la que provoca cambios y activación fisiológica de tipo externo (sudoración, dilatación pupilar, temblor, tensión muscular, palidez facial, entre otros), e involuciones o parcialmente soluciones (palpitaciones, temblor, vómitos, desmayos, entre otros). Existe una última dimensión denominada autoconfianza que se relaciona con la creencia de que los sujetos están preparados para la situación a la que se enfrentan (López Torres, Torregrosa, \& Roca, 2007; Tsopani, Dallas, \& Skordills, 2011). La autoconfianza sería prácticamente lo opuesto a la 
ansiedad cognitiva (Moreno, Moreno-Murcia, González, Pulido, \& Cervelló, 2012). Para Zarauz y Ruiz-Juan (2013) los deportistas de éxito presentan puntuaciones altas en autoconfianza y moderadas en ansiedad cognitiva y somática.

La ansiedad precompetitiva puede ser positiva o indiferente hacia la ejecución deportiva, dependiendo de aspectos como la personalidad del deportista, la dificultad de la tarea o el nivel de habilidad del individuo (Brandao, 1995; Cervelló, Santos-Rosa, Jiménez, Nerea, \& García, 2002). La investigación efectuada en el campo de la ansiedad competitiva en el deporte ha mostrado la existencia de una serie de elementos, tanto personales como de situaciones, relacionados con la aparición de la ansiedad previa a la competición (Cervelló et al., 2002). Desde una perspectiva cognitivo-afectiva los elementos responsables de la aparición de la ansiedad no serán sólo personales, sino que también influyen aspectos tales como el tipo de deporte o la complejidad de la tarea (Burton, 1998). Centrándonos en el tiro deportivo en su modalidad de precisión, entre los factores que pueden estar relacionados con la ansiedad están: objetivos individuales inapropiados para la competición, nivel de los rivales con los que se compite, material individual inadecuado, la no familiarización con el contexto. En este sentido, el ambiente en el que se encuentra compitiendo el deportista (Arbinaga \& Caracuel, 2005), el tipo y características de la prueba a la que se enfrenta (Jaenes, 2000), así como el tipo de deporte (Martens, et al., 1990), la experiencia en competición (Mellalieu, Hanton \& O'Brien, 2004) e, incluso, la percepción del deportista de ir o no adecuadamente entrenado a competir (Gutiérrez, Estévez, García \& Pérez, 1997), el nivel de la competición y por las características de cada disciplina deportiva (Kais y Raudsepp, 2004; Mellalieu, et al., 2004) pueden influir en la intensidad de la ansiedad experimentada y la percepción direccional de la misma.

En esta línea, estudios realizados con jugadores de bádminton (Eubank, Smith, \& Smethurst, 1995) y gimnastas (Jones, Swain, \& Hardy, 1993) aseveran que mantener bajos niveles de ansiedad con una percepción direccional facilitadora podría ser la respuesta más adecuada para predecir un óptimo rendimiento en competición. No obstante, publicaciones recientes indican que la interpretación facilitadora de los síntomas de ansiedad son consecuencia de unos niveles bajos de los mismos y posiblemente combinado con altos niveles de autoconfianza (Lundqvist, Kenttä, \& Raglin, 2010; Mullen, Lane, \& Hanton, 2009).

En un estudio reciente con golfistas coreanos de diferente nivel se constató que a pesar de que ambos grupos tenían elevados niveles de ansiedad somática antes y durante la competición, los jugadores de élite tenían niveles inferiores de ansiedad cognitiva y niveles superiores de autoconfianza respecto a los de menor nivel competitivo antes y durante la competición (Kim, Chung, Park, \& Shin, 2009). Por otra parte, Burton (1988) encontró una relación inversamente lineal entre la ansiedad cognitiva y el rendimiento y una relación directamente lineal entre éste y la autoconfianza.

En este marco de trabajo, y ante la escasa información que se dispone de un deporte como el tiro deportivo, se plantea como objetivo analizar los niveles de estados de ánimo y ansiedad precompetitiva, según el nivel o categoría de los deportistas. Como hipótesis de partida, se espera encontrar un perfil iceberg en los estados de ánimo, siendo este más pronunciado en los tiradores de primera categoría, que los de segunda y tercera. Asimismo se espera encontrar puntuaciones altas en autoconfianza y moderadas en ansiedad cognitiva y ansiedad somática, siendo más acuciadas estas diferencias en los tiradores de primera que en las otras dos categorías.

\section{2.- MATERIAL Y MÉTODO}

\section{Participantes}

El diseño de este trabajo es descriptivo y correlacional de carácter transversal en el que participaron 183 deportistas con edades comprendidas entre 26 y 59 años $(M=41.14$; DT=6.43); 179 eran hombres $(97.8 \%)$ y solamente cuatro mujeres $(2.2 \%)$, procedentes de las provincias de Almería, Granada y Málaga. Para la clasificación de los tiradores por categorías se siguen los criterios de la Real Federación Española de Tiro Olímpico, participando: 33 eran de primera categoría (18.0\%), 78 de segunda $(42.7 \%)$ y 72 de tercera $(39.3 \%)$. 
La muestra fue de 1.008 personas ( 498 varones $=49.4 \%$ y 510 mujeres $=50.6 \%$ ). El rango de edad estuvo comprendido entre los 15 y 87 años $(M=36.57$; $D T=17.05)$. Los componentes de la muestra dieron su consentimiento para participar en el estudio.

\section{Instrumentos}

Inventario de Ansiedad Competitiva-2 Revisado (CSAI-2R). Se utilizó la versión adaptada al contexto español por Andrade, Lois, y Arce (2007) del Revised Competitive State Anxiety Inventory-2 (CSAI-2R) de Cox, Martens, y Russell (2003). Este instrumento consta de tres subescalas: ansiedad cognitiva, ansiedad somática y autoconfianza. La primera y tercera se componen por cinco ítems puntuables de 1 (nada) a 4 (mucho) con puntuación total entre 5 y 20. La segunda contiene seis ítems, con puntuación total entre 6 y 24. En la presente investigación la consistencia interna hallada fue: ansiedad cognitiva, $\alpha=.78$; ansiedad somática, $\alpha=.81$; autoconfianza, $\alpha=.87$.

Profile of Mood States (POMS). Se utilizó la versión adaptada y validada al contexto español por Balaguer, Fuentes, Meliá, García-Merita, \& Pérez (1994) del original Profile of Mood States de McNair et al. (1971). Consta de cuatro dimensiones negativas: tensión, depresión, hostilidad y fatiga; y una positiva, vigor. En esta investigación se emplean la subescala positiva de vigor, compuesta por ocho ítems que sugieren un estado de ánimo de vigorosidad, euforia y energía elevada: animado, activo, enérgico, alegre, alerta, lleno de energía, despreocupado, vigoroso; y subescala negativa de fatiga, que corresponde a un estado de abatimiento (desgaste), inercia y bajo nivel de energía y a la que se refieren los siguientes siete ítems: agotado, apático, fatigado, exhausto, espeso, sin fuerzas y cansado. Los datos se recogieron en una escala tipo Likert con valores que oscilan desde 0 (nada) a 4 (muchísimo). En la presente investigación la consistencia interna hallada fue: vigor, $\alpha=.72$; fatiga, $\alpha=$ .87 .

\section{Procedimiento}

Los datos fueron obtenidos en una fase clasificatoria para el Campeonato de Andalucía de Tiro Deportivo, celebrada los días 28 y 29 de septiembre de 2013, en el Campo de Tiro de la Asociación de Clubes de Tiro Olímpico Almerienses (ACTOA), situado en la provincia de Almería. Para la recogida de datos a los tiradores se cuenta con la autorización de los directivos del Club y se pasaron los correspondientes consentimientos informados para aquellos deportistas que quisieran participar en la investigación. La aplicación de las escalas CSAI-2R y POMS se efectuó por líneas de tiro de 15 tiradores, unos 30 minutos antes de comenzar la competición, al entrar a cámara de llamada. Para la clasificación de los tiradores por categorías se utilizó la clasificación de la Real Federación Española de Tiro Olímpico (RFEDETO): primera, segunda y tercera categoría, según las puntaciones obtenidas por los tiradores en las competiciones organizadas por la federación.

Las puntuaciones obtenidas son facilitadas por la organización y corresponden a las cinco primeras series de cada tirador, es decir, serie de prueba y cuatro primeras de competición. Para calcular la dispersión de los impactos se fotografían todas las series con una cámara digital y se miden las distancias de cada impacto respecto de la horizontal y la vertical que pasan por el centro de la diana, obteniendo un $\left(d x_{n}\right)$ y un $\left(d y_{n}\right)$ para cada disparo. Dicho valor se adapta a una escala de 1 al 10. Cada par de datos $\left(d x_{n}, d y_{n}\right)$ determinan un punto en el plano, a partir del cual se deduce la distancia al centro:
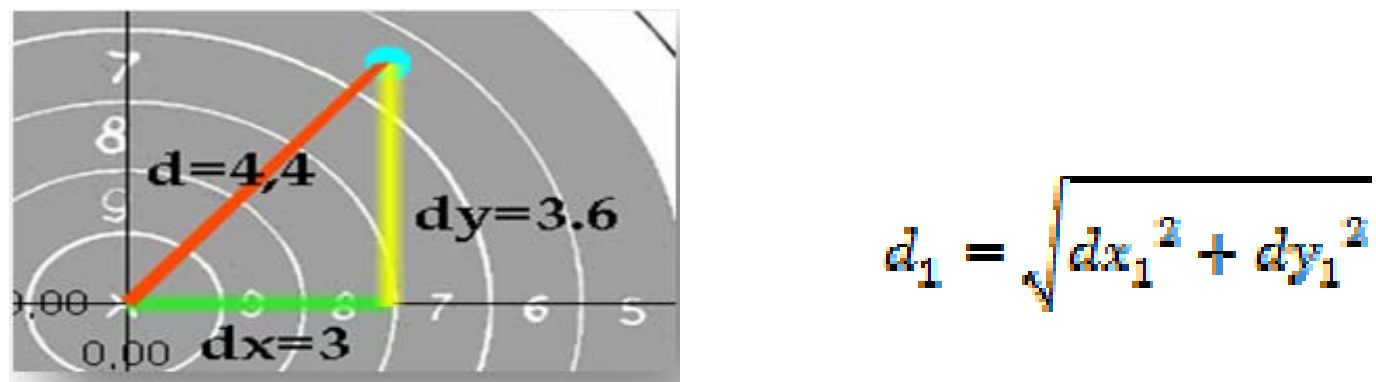

Figura 1.- Procedimiento seguido para el cálculo de la dispersión de los impactos 
El valor de cada " $d_{n}$ " se corresponde con la puntuación obtenida en dicho impacto [inversa según la fórmula $p_{n}=10-\operatorname{Int}\left(d_{n}\right)$ ] lo que sirve como elemento de comprobación y depuración de errores. Se calculan los valores promedio de los valores $d x_{n}$ y $d y_{n}$ y sus desviaciones estándar. Este par de datos $(d x, d y)$ de cada conjunto, así obtenidos, son las coordenadas del centro de la agrupación, desde la que se deducen los datos de cuadrante, distribución horizontal vertical, distribución derecha izquierda y alineaciones vertical, horizontal o centrada. La distancia al centro $d$ del par promedio $(d x, d y)$ es el valor absoluto que establece el nivel de centrado de cada tirador. La desviación estándar de los valores $\left(d x_{n}\right)$ y $\left(d y_{n}\right)$ determinan la dispersión. El resultado de multiplicar las desviaciones estándar (Sx) con la (Sy) muestra el dato absoluto de dispersión de cada conjunto.

La Figura 1 representa gráficamente la recogida real de impactos efectuados por la muestra. En su punto A, representa a través de puntos rojos los 4575 impactos realizados por la totalidad de la muestra objeto de estudio. En su punto B representa los 25 impactos correspondientes a un tirador, con un rombo rojo representando el punto medio de los impactos. El punto $\mathrm{C}$ representa la agrupación del $7.2 \%$ de la muestra, que son los tiradores zurdos. Finalmente el punto D representa los tiradores cuya mano dominante es la izquierda (7.2\%), agrupan los disparos a la derecha, y los tiradores que su mano dominante es la derecha $92.8 \%$ agrupan a la izquierda, el número uno muestra el punto medio de los impactos de los tiradores de primera categoría, el número dos el punto medio de los impactos de los tiradores de segunda, y el tres, el punto medio de los impactos de los tiradores de tercera.

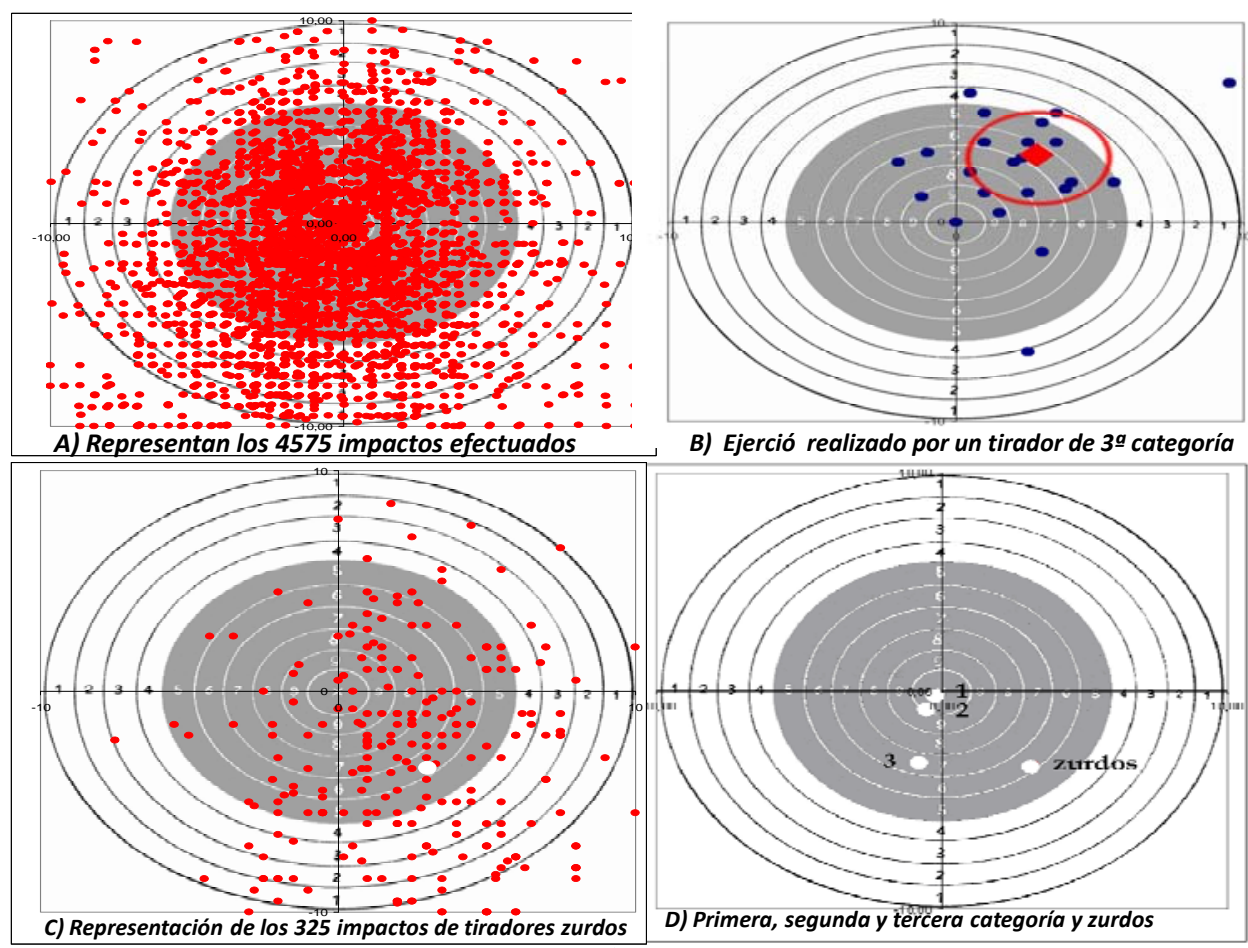

Figura 2.- Representación de la relación entre las variables psicológicas objeto de estudio y los efectos fisiológicos

\section{Análisis estadísticos de los datos}

Los análisis de ítems, homogeneidad, consistencia interna de cada subescala (alfa de Cronbach), correlaciones bivariadas y análisis multivariante de la varianza (MANOVA) se realizaron con SPSS 17.0. El análisis de la dispersión de los impactos en las dianas se efectuó mediante un software específico para ello. 


\section{3.- RESULTADOS}

\section{Análisis descriptivo}

Los estadísticos de las dimensiones estudiadas se pueden observar en la Tabla 1. En el caso de los estados de ánimo, la puntuación del vigor es muy superior a la de fatiga. En la ansiedad competitiva, se comprueba que la población estudiada presentó un puntaje mucho más alto en autoconfianza que en ansiedad, tanto somática como cognitiva. También se muestran las correlaciones entre las dimensiones estudiadas. Se puede destacar la alta correlación positiva entre el vigor y la autoconfianza. Asimismo, la fatiga correlacionó positiva y significativamente con la ansiedad, sobre todo con la somática. En la parte negativa, reseñar la correlación del vigor con la fatiga y la ansiedad somática; también la autoconfianza correlacionó negativa y significativamente con la ansiedad somática y la fatiga.

\section{Tabla 1}

Estadísticos descriptivos y correlaciones bivariadas entre las subescalas del POMS y CSAI-R2.

\begin{tabular}{|c|c|c|c|c|c|c|c|}
\hline Subescalas & $M$ & $D T$ & 1 & 2 & 3 & 4 & 5 \\
\hline Vigor & 19.43 & 5.35 & & $-.22 * *$ & $.55^{* *}$ & .09 & $-.22 * *$ \\
\hline Fatiga & 3.97 & 4.63 & & & $-.20 * *$ & $.24 * *$ & $.40 * *$ \\
\hline Autoconfianza & 15.54 & 2.88 & & & & -.05 & $-.34 * *$ \\
\hline Ansiedad cognitiva & 7.34 & 2.56 & & & & & $.33 * *$ \\
\hline Ansiedad somática & 9.87 & 2.74 & & & & & \\
\hline
\end{tabular}

${ }^{*} p<.05 ; * *<.01 ; M=$ puntuación media; $D T=$ desviación típica

La Figura 2 describe la muestra global apreciándose un claro perfil iceberg en las escalas de estado de ánimo, con niveles de vigor alto y fatiga baja. Los datos aportados por el CSAI-2R muestran niveles altos de autoconfianza y moderados de ansiedad cognitiva y somática.

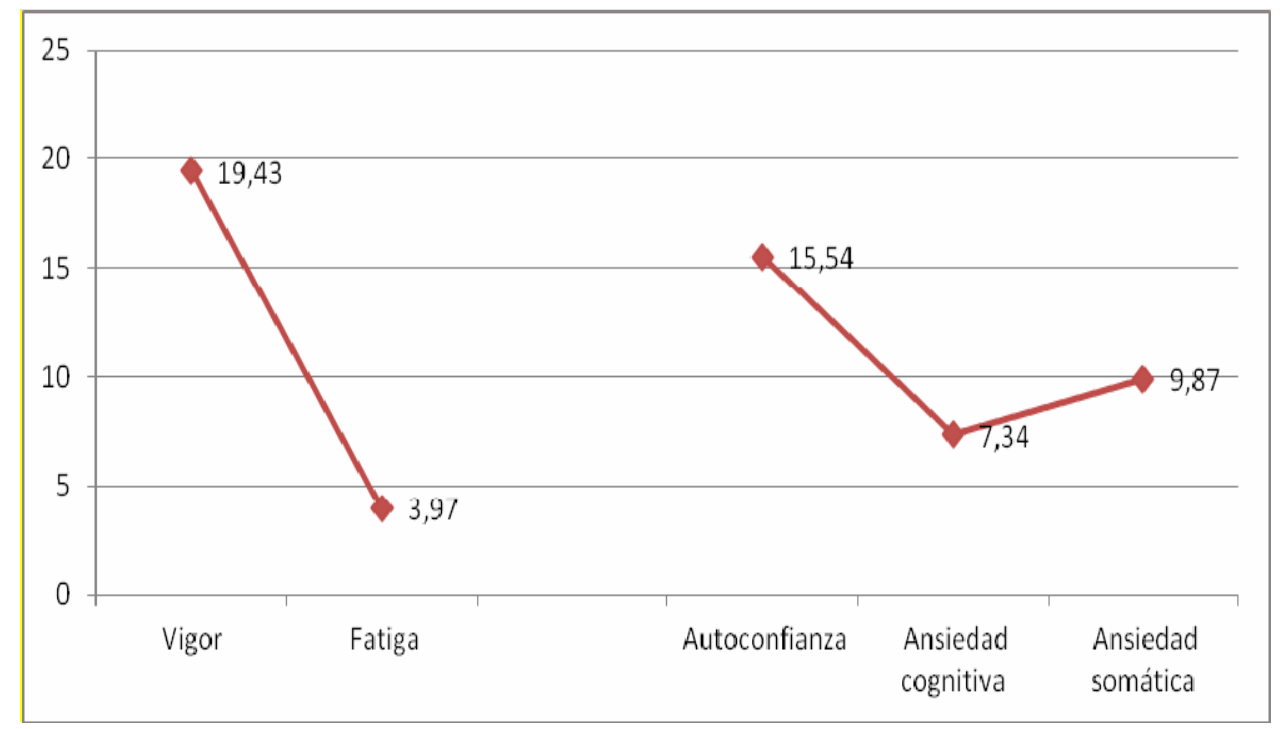

Figura 3.- Representación grafica de los valores de las variables psicológicas correspondientes a la muestra (POMS y CSAI-2R)

Como se puede apreciar en la Figura 3, el perfil icebeg descrito por el POMS, la escala vigor presenta valores constantes y descendentes a medida que desciende el nivel deportivo por el contrario la escala fatiga muestra un comportamiento inverso salvo en los tiradores de tercera categoría. 


\section{Análisis multivariante de la varianza}

Para analizar los efectos de interacción del nivel (o categoría) de disparo alcanzado por el tirador sobre los constructos analizados, se realizó un análisis multivariante de la varianza (MANOVA) en el que la variable independiente fue el nivel del tirador y las dependientes las subescalas del POMS y CSAI-R2. En el contraste de comparaciones múltiples post hoc se realizó la prueba de Bonferroni.

La homogeneidad de la covarianza se examinó mediante el test $\mathrm{M}$ de Box; se rechazó la hipótesis nula de ajuste de los datos $(\mathrm{M}$ de $\mathrm{Box}=53.03, F=1.68, p<.001)$. Se siguieron las sugerencias de Tabachnick y Fidell (2007) de usar la Pillai's Trace en lugar de la Lamba de Wilks para evaluar la significación multivariada de efectos principales y de las interacciones. El contraste multivariado demostró diferencias significativas en función del nivel de los tiradores (Pillai's Trace $=$ $.01, F_{(10,354)}=1.86, p=.049$, tamaño del efecto $=.05$; potencia observada $\left.=.85\right)$.

Tabla 2

Análisis multivariante (efectos intersujetos según categoría) en función de las subescalas del POMS y CSAI-R2.

\begin{tabular}{lccccccccccc}
\hline & $\begin{array}{c}\text { Primera } \\
\text { Categoría } \\
(n=33)\end{array}$ & $\begin{array}{c}\text { Segunda } \\
\text { Categoría } \\
(n=78)\end{array}$ & $\begin{array}{c}\text { Tercera } \\
\text { Categoría } \\
(n=72)\end{array}$ & & & & & \\
\hline & $M$ & $D T$ & $M$ & $D T$ & $M$ & $D T$ & $F$ & $p$ & $d$ & $\begin{array}{c}\text { Potencia } \\
\text { observada }\end{array}$ \\
\hline Vigor & 21.42 & 4.37 & 19.18 & 5.54 & 18.78 & 5.40 & 2.97 & .054 & .03 & .57 \\
Fatiga & 3.39 & 4.15 & 4.23 & 4.84 & 3.96 & 4.64 & .38 & .687 & .00 & .11 \\
Autoconfianza & 16.67 & 2.33 & 15.46 & 2.94 & 15.10 & 2.94 & 3.50 & .032 & .04 & .65 \\
Ansiedad cognitiva & 7.06 & 2.30 & 7.00 & 2.33 & 7.83 & 2.85 & 2.25 & .108 & .02 & .45 \\
Ansiedad somática & 9.00 & 1.64 & 9.60 & 2.58 & 10.56 & 3.16 & 4.43 & .013 & .05 & .76 \\
\hline
\end{tabular}

Nota. $p$ es significativo al nivel $<.05$

Las pruebas intersujetos mostraron diferencias significativas en la autoconfianza $[F(2180)=$ $3.50, p=.032]$ y en la ansiedad somática $[F(2180)=3.43, p=.013]$ (Tabla 2). En el caso de la autoconfianza, las puntuaciones medias más altas corresponden a los de mejor nivel (primera) y van descendiendo las puntuaciones a medida que desciende el nivel. Se pueden observar los resultados de forma gráfica en la Figura 4. Las pruebas a posteriori (Bonferroni) arrojaron diferencias significativas entre los de primer y los de tercer nivel $(p=.028)$. En la ansiedad somática ocurre lo contrario, ya que las puntuaciones medias más altas corresponden a los de menor nivel (tercera) y más bajas a los de mejor niveles (primeros) (Figura 4). La prueba de Bonferroni mostró diferencias significativas entre los de nivel de tercera y los de primera $(p=.020)$.

\section{4.- DISCUSIÓN}

El objetivo de este trabajo fue analizar el estado de ánimo y la ansiedad precompetiva según el nivel o categoría de tiradores de precisión. Como hipótesis de partida, se esperaba hallar un perfil iceberg en los estados de ánimo, más pronunciado en los tiradores de primera categoría, que los de segunda y tercera.

Los datos encontrados en las variables del estado de ánimo, en las tres categorías, coinciden con el denominado perfil "Iceberg" en el que la escala vigor se sitúa por encima de fatiga. Por lo que se cumple la hipótesis de partida. Este perfil predispondrá al deportista a obtener un elevado rendimiento competitivo que se correspondería con un estado de ánimo óptimo (Morgan, 1980a, 1980b; O'Connor, 2006). En cada categorías (Figura 4), las puntuaciones de vigor presentan una evolución constante y descendente de un modo prácticamente lineal -a mayor nivel deportivo mayor vigor-; por el contrarío, la fatiga presenta una evolución inversa al vigor, es decir, a menor nivel 
deportivo más fatiga, salvo en la muestra de tercera categoría, que no se comporta de un modo lineal, posiblemente asociado a un sesgo muestral. Estos datos están en la línea los hallados por Cox (2008) quien observó como los deportistas de mayor éxito obtenían un perfil de estados de ánimo más saludable, frente a aquellos menos exitosos o con una población control.

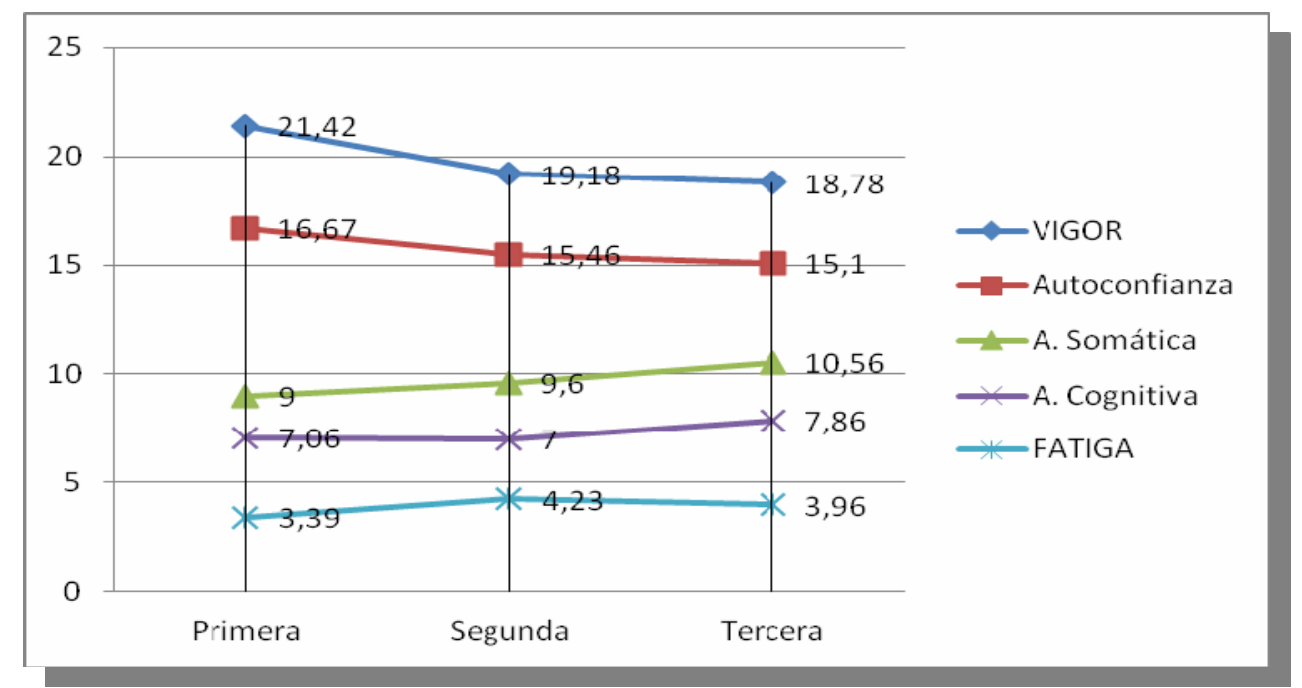

Figura 4.- Representación grafica de los valores estados de ánimo (POMS), escalas de vigor y fatiga, y de los valores autoconfianza, ansiedad cognitiva y ansiedad somática (CSAI-2R), correspondientes a los tres niveles de tiradores

En relación con los niveles de autoconfianza y ansiedad, se cumple la segunda hipótesis de este trabajo. En el caso de la ansiedad precompetitiva, se comprueba que la población estudiada presentó un puntaje superior en autoconfianza que en ansiedad, tanto somática como cognitiva, siendo en esta ultima variable en la que menos puntúan las tres categorías. Estos resultados van en la línea de los expuestos por Moreno et al. (2012), quienes sostienen que la autoconfianza sería prácticamente lo opuesto a la ansiedad cognitiva. La representación grafica de las variables de ansiedad por categorías (Figura 4) muestra datos similares a los de Burton (1988), quien halló una relación inversamente lineal entre la ansiedad cognitiva y el rendimiento y una relación directamente lineal entre éste y la autoconfianza. También coinciden con el estudio de Kim, et al., (2009), quienes sostuvieron que los jugadores de élite tenían niveles inferiores de ansiedad cognitiva y niveles superiores de autoconfianza respecto a los de menor nivel competitivo antes y durante la competición. La ansiedad somática muestra una evolución similar a la ansiedad cognitiva e inversa a la autoconfianza. Estos resultados van en la línea Ruiz y Rodríguez (2013), quienes encuentran relación inversa y negativa entre la ansiedad y el rendimiento deportivo en tiro.

Respecto a las correlaciones entre las dimensiones estudiadas, destaca la alta correlación positiva entre el vigor y la autoconfianza. Resulta lógica esta correlación si se tiene en cuenta que el vigor es el afecto o estado de ánimo positivo (Watson \& Clark, 1994, 1997) y la autoconfianza, las creencias de los sujetos de estar preparados para la situación a la que se enfrentan (López Torres et al., 2007; Tsopani, et al., 2011), en este caso, tiro de precisión.

Por otro lado, la fatiga correlacionó positivamente con la ansiedad somática. Atendiendo a las explicaciones de Watson y Clark (1994, 1997), apuntadas en la introducción, así como las de Cheng, et al., (2009), Hardy, et al., (1996), Martens et al., (1990), es comprensible que se produzcan alteraciones y síntomas corporales asociados al sistema nervioso autónomo a la hora de enfrentarse a las situaciones de estrés de esta competición.

Respecto a la agrupación de los impactos, los tiradores zurdos tienen tendencia agrupar los impactos a la derecha de la diana, mientras que los diestros tienen ligera tendencia agrupar a la izquierda, estos efectos aparentemente se deben a cuestiones biomecánicas. En las agrupaciones de los 
impactos por niveles o categorías deportivas se observa una tendencia a la izquierda y hacia abajo, siendo mayor la centralización en los tiradores de primera y mayor dispersión en los tiradores de tercera. Este patrón, representado en la Figura 2D, se muestra constante en los tiradores diestros.

Para finalizar, los resultados encontrados en este estudio van en la línea de los mostrados por Burton (1988), quien encontró una relación inversamente lineal entre la ansiedad cognitiva y el rendimiento y una relación directamente lineal entre éste y la autoconfianza. En el presente estudio se encuentra una relación directamente lineal entre el rendimiento y el vigor y la autoconfianza y una relación inversamente lineal entre éste y la ansiedad somática. Se presentan pequeñas desviaciones en la linealidad en el caso de la ansiedad cognitiva, en la segunda categoría y en la fatiga en la tercera categoría, quizás asociado a algún tipo de sesgo muestral.

En las variables del estado de ánimo aparece un claro "perfil iceberg". Respecto a la dispersión de los impactos se observar una descentralización con tendencia hacia la izquierda y hacia abaja siendo mayor la dispersión a medida que desciende la categoría.

La principal limitación de este estudio es que al tratarse de un deporte totalmente amateur los objetivos de los tiradores no resultan claros. Pues si bien el objetivo del campeonato es clasificarse para el Campeonato de Andalucía, compiten tiradores que no les interesa asistir al mismo; esta puede ser la principal causa de que la grafica no sea totalmente lineal en las variables ansiedad cognitiva segunda categoría y fatiga en la tercera categoría.

La principal fortaleza de esta investigación es que aporta datos relevantes respecto a los efectos de los estados de ánimo y la ansiedad precompetitiva sobre el rendimiento en el tiro deportivo, así como el afecto de estas variables psicológicas en las distintas categorías competitivas. Cabe reseñar que el Tiro ha sido incluido en el Programa Olímpico desde la celebración de los primeros Juegos Olímpicos de la era moderna. A pesar de ello son escasas las investigaciones sobre este deporte debido, sobre todo, a la dificultad de acceso a muestras relevantes. Ello otorga importancia a la contribución de este estudio a la literatura científica. Un punto destacable de este trabajo es que la recogida de datos se realizara minutos antes de la competición, lo cual incrementa la precisión de las variables psicológicas estudiadas y su relaciones.

\section{5.- REFERENCIAS}

Abenza, L., Olmedilla, A., Ortega, E., Ato, M., \& García-Más, A. (2010). Análisis de la relación entre el estado de ánimo y las conductas de adherencia en deportistas lesionados. Anales de Psicología, 26(1), 159-168.

Andrade, E. M., Lois, G., \& Arce, C. (2007). Propiedades psicométricas de la versión española del Inventario de Ansiedad Competitiva CSAI-2R en deportistas. Psicothema, 19, 150-155.

Andrade, E., Arce, C., Armental, J., Rodríguez, M., \& De Francisco, C. (2008). Psicothema, 20, 630-635.

Arbinaga Ibarzábal, F, \& Caracuel Tubío, J. C. (2005). Precompetición y ansiedad en fisicoculturistas. Revista de Psicología del Deporte, 14, 195-208.

Arbinaga, I. (2013). Fisicoculturismo: diferencias de sexo en el estado de ánimo y la ansiedad precompetiva. Revista de Psicología del Deporte, 22, 353-360.

Arruza, J. (1995). Estado de ánimo, esfuerzo percibido y frecuencia cardiaca. Un estudio aplicado al entrenamiento de Judo. Bilbao: Servicio Editorial U.P.V./E.H.U.

Arruza, J., Arribas, S., Otaegi, O., González, O., Irazusta, S., \& Ruiz, J. M. (2011). Percepción de competencia, estado de ánimo y tolerancia al estrés en jóvenes deportistas de alto rendimiento. Anales de Psicología, 27, 536-543.

Arruza, J., Balagué, G., \& Arrieta, M. (1998). Rendimiento deportivo e in-fluencia del estado de ánimo, de la dificultad estimada, y de la autoeficacia en la alta competición. Revista de Psicología del Deporte, 7, 193204.

Balaguer, I., Fuentes, I., Meliá, J. L., García-Merita, M. L., \& Pérez-Recio, G. (1994). El perfil de los estados de ánimo (POMS): Baremos para estudiantes valencianos y su aplicación en el contexto deportivo. Revista de Psicología del Deporte, 4, 39-52.

Brandao, R. F. (1995). Ansiedade em atletas. Movimento, 1, 24-27. 
Burton, D. (1988). Do anxious swimmers swin slower? reexamining the elusive anxiety-performance relationship. Journal of Sport and Exercise Psychology, 10, 45-61.

Cervelló, E., Santos-Rosa, F. J., Jiménez, R., Nerea, A., \& García, T. (2002). Motivación y ansiedad en jugadores de tenis. Motricidad, 9, 141-161.

Cheng, W. N., Hardy, L., \& Markland, D. (2009). Toward a three-dimensional conceptualization of performance anxiety: rationale and initial measurement development. Psychology of Sport and Exercise, 10, 2714278.

Cox, R. H., Martens, M. P., \& Russell W. D. (2003). Measuring anxiety in athletics: The revised Competitive State Anxiety Inventory-2. Journal of Sport and Exercise Psychology, 25, 519-533.

Cox, R.H. (2008). Psicología del deporte: conceptos y sus aplicaciones. Madrid: Editorial Médica Panamericana.

De la Vega, R., Ruiz Barquín, R., García Adrianzén, G. D, \& Del Valle, S. (2011). El estado de ánimo precompetitivo en un equipo de fútbol profesional: un estudio entre jugadores titulares y suplentes. Cuadernos de Psicología del Deporte. 11, 107-117.

De la Vega, R., Ruiz Barquín, R., García-Mas, A., Balagué, G., Olmedilla, A., \& Del Valle, S. (2008). Consistencia y fluctuación de los estados de ánimo en un equipo de fútbol profesional durante una competición de play off. Revista de Psicología del Deporte, 17(2), 241-251.

Eubank, M. R., Smith, N. C. \& Smethurst, C. J. (1995). Intensity and direction of multidimensional competitive state anxiety: Relationships to performance in racket sports. Journal of Sport Sciences, 13(1), 52-53.

Eysenck, M. W., \& Calvo, M. G. (1992). Anxiety and performance. The processing efficiency theory. Cognition and Emotion, 6, 409-434.

Gutiérrez Calvo, M., Estévez, A., García Pérez, J., \& Pérez Hernández, H. (1997). Ansiedad y rendimiento atlético en condiciones de estrés: efectos moduladores de la práctica. Revista de Psicología del Deporte, $6(2), 27-46$.

Hardy, L., Jones, G., \& Gould, D. (1996). Understanding psychological preparation in sport: theory and practice of elite performers. Chichester, UK: Wiley.

Jaenés Sánchez, J. C. (2000). Estado emocional y conducta deportiva: Ansiedad competitiva en corredores de maratón. Tesis Doctoral. Universidad de Sevilla.

Jones, G., Swain, A., \& Hardy, L. (1993). Intensity and direction dimensions of competitive state anxiety and relationships with performance. Journal of Sports Sciences, 11, 525-532.

Kais, K., \& Raudsepp, L. (2004). Cognitive and somatic anxiety and self-confidence in athletic performance of beach volleyball. Perceptual \& Motor Skills, 98, 439-449.

Kim, K. J.; Chung, J. W., Park, S., \& Shin, J. T. (2009). Psychophysiological Stress Response during Competition between Elite and Non-elite Korean Junior Golfers. International Journal of Sports Medicine, 30, 503-508.

Liebert, R. M. \& Morris, L. W. (1967). Cognitive and emotional components of test anxiety: a distinction and some initial data. Psychology Reports, 20, 975-978.

López-Torres, M., Torregrosa, M., \& Roca, J. (2007). Características del "flow", ansiedad y estado emocional en relación con el rendimiento de deportistas de elite. Cuadernos de Psicología del Deporte, 7, 25-44.

Lundqvist, C., Kenttä, G., \& Raglin, J.S. (2010). Directional anxiety responses in elite and sub-elite young athletes: intensity of anxiety symptoms matters. Scandinavian Journal of Medicine \& Science in Sports. ([Epub ahead of print]).

Martens, R., Vealey, R. S., \& Burton, D. (1990). Competitive Anxiety in Sport. Champaign, IL: Human Kinetics.

McNair, D., Loor, M., \& Droppleman, L. (1971). Manual for the Profile of Mood States, San Diego CA: Educational and Industrial Testing Service.

Mellalieu, S., Hanton, S., \& O'Brien, M. (2004). Intensity and direction of competitive anxiety as a function of sport type and experience. Scandinavian Journal of Medicine and Science in Sports, 14, 326-334.

Moreno, C., Moreno-Murcia, J. A., González, I., Pulino, J. J., \& Cervello, M. (2012). Ansiedad estado precompetivo en judocas. Revista de Artes Marciales Asiáticas. 7, $26-43$.

Moreno, J., Parrado, E., \& Capdevila, L. (2013). Variables de la frecuencia cardiaca y perfiles psicofisiológicos en deporte de equipo de alto rendimiento. Revista de Psicología del Deporte, 22, 343-352. 
Morgan, W. P. (1980a). Test of champions. Psychology Today, Julio, 92-99.

Morgan, W. P. (1980b). The trait psychology controversy. Research Quarterly for Exercise and Sport, 51, 5076.

Mullen, R., Lane, A., \& Hanton, S. (2009). Anxiety symptom interpretation in highanxious, defensive highanxious, low-anxious and repressor sport performers. Anxiety, Stress \& Coping, 22(1), 91-100.

O’Connor, P. J. (2006). Mental Energy: Assessing the Mood Dimension. Nutrition Reviews, 64(7), S7-S9.

Rey, M., Blasco, T., \& Borrás, F. (2000). Efectos de un procedimiento de inducción de estados de ánimo sobre la autoeficacia. Anales de Psicología, 16(1), 33-40.

Ruiz, R., \& Rodríguez, J. M. (2013). El estado de flow y la ansiedad en la modalidad de tiro IPSC. Actas del XIV Congreso Andaluz de la Actividad Física y el Deporte. Huelva (España).

Tabachnick, B. G., \& Fidell, L. S. (2007). Using Multivariate Statistics (5 a ed.). New York: Allyn and Bacon.

Torres-Luque, G., Hernández-García, R., Olmedilla, A., Ortega, E., \& Garatachea, V. (2013). Fluctuaciones del Perfil de Estado de Ánimo (POMS) en un periodo competitivo de judokas de élite. Revista de Psicología del Deporte. 22, 313-320.

Tsopani, D., Dallas, G., \& Skordilis, E.K. (2011). Competitive state anxiety and performance in young female rhythmic gymnasts. Perceptual and Motor Skills, 112(2), 549-560.

Watson, D., \& Clark, L. A. (1994). Emotions, moods, trais and temperaments: conceptual distinctions and empirical findings. En P. Ekman, \& R. Davidson (Eds.), The nature of emotion (pp. 98-93). Oxford: Oxford University Press.

Watson, D., \& Clark, L. A. (1997). Meassurement and mismeasurement of mood: recurrent and emergent issues. Journal of Personality Assesment, 68, 267-296.

Zarauz, A., \& Ruiz-Juan, F. (2013). Variables predictoras de la ansiedad en atletas veteranos españoles. Retos. Nuevas tendencias en Educación Física, Deporte y Recreación. 23, 29-32.

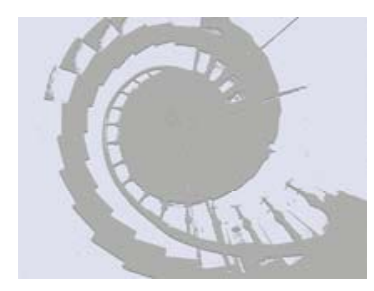

